\title{
Wind turbine wake properties : Comparison between a non-rotating simplified wind turbine model and a rotating model
}

\author{
S. Aubrun ${ }^{1}$, S. Loyer ${ }^{1}$, P. E. Hancock ${ }^{2}$ and P. Hayden ${ }^{2}$ \\ 1 Laboratoire PRISME, Université d'Orléans \\ 8 rue Léonard de Vinci F-45072 Orléans Cedex 2, France \\ 2 EnFlo Laboratory, University of Surrey, Surrey, UK
}

\begin{abstract}
Experimental results on the wake properties of a non-rotating simplified wind turbine model, based on the actuator disc concept, and a rotating model, a three-blade wind turbine, are presented. Tests were performed in two different facilities, one providing a nominally Decaying Isotropic Turbulent inflow (turbulence intensity of $4 \%$ at rotor disc location) and one providing a neutral atmospheric boundary layer above a moderately rough terrain at a geometric scale of $1: 300$ (determined from the combination of several indicators), with $13 \%$ of turbulence intensity at hub height). The objective is to determine the limits of the simplified wind turbine model to reproduce a realistic wind turbine wake. Pressure and high-order velocity statistics are therefore compared in the wake of both rotor discs for two different inflow conditions in order to quantify the influence of the ambient turbulence. It has been shown that wakes of rotating model and porous disc developing in the modelled atmospheric boundary layer are indistinguishable after 3 diameters downstream of the rotor discs, whereas few discrepancies are still visible at the same distance with the Decaying Isotropic Turbulent inflow.
\end{abstract}

Key words: Porous disc, Wind turbine, Wake, Homogeneous and Isotropic Turbulent flow, Atmospheric Boundary Layer flow, wind tunnel

\section{Introduction}

In order to study the wind turbine wake and its eventual interactions with neighbouring wind turbines, several numerical and physical modelling approaches are used. Some model the wind turbine with the simplest model, which is the actuator disc concept, adding a drag source (i.e. pressure loss) within the surface swept by the blades (exemples for numerical [1] and physical applications $[5,2,3,11,10])$. Some use the Blade Element Momentum Theory, which takes into account the blade rotation effect on the wake and the aerodynamic features of the blades $[13,6]$. Some use Reynolds-Averaged-Navier-Stokes simulation 
or Large-Eddy-Simulation to compute the steady and unsteady flows through modelled or real rotors $[16,15,19]$. In a wind resource assessment context, the latter one is not practical enough to be used since the computation times are extremely long, even if it delivers the better results with respect to the instationary process. Consequentely, RANS simulation is still the most attractive to model the far wake, according to its simplicity of implementation and short computation time. On the other hand, the issue is that it is difficult to assess the errors induced by the absence of blades and associated rotation momentum on the wake development. Furthermore, the level of turbulence intensity encountered in the atmospheric incoming flow plays a role on this issue : First, the higher the turbulence intensity is, the faster the spectral signature of the blades disappears in the wake and the faster the tangential velocity induced by the rotational momentum is overwhelmed in ambiant velocity fluctuations. These observations need to be quantified. In this context, the present study compares the wake properties of a model of a three-blade rotating wind turbine [14] and of a porous disc made of metallic mesh, generating the same velocity deficit as the wind turbine. Both models are tested in a modelled atmospheric boundary layer flow and in a Decaying Isotropic Turbulent flow in order to see the influence of the approach flow conditions. The velocity statistics are compared between both models in order to determine whether the far wake state is reached at $x=3 D$. Here, the far wake is defined by the self-similarity of the velocity deficit and turbulence intensity profiles downstream of models (Gaussian-type distributions). The spectral content of the flow is also studied in order to determine whether the blade signature in the wake is still visible at this location.

\section{Experimental set-up}

In this study, all measurements are performed in the closed circuit wind tunnel of the PRISME laboratory (Fig. 1). This facility is equipped with two test sections. The main one is $2 \mathrm{~m}$ high, $2 \mathrm{~m}$ wide and $5 \mathrm{~m}$ long and is used in the present study to generate Decaying 'Isotropic' Turbulent flow. The second one is $5 \mathrm{~m}$ high, $5 \mathrm{~m}$ wide and $20 \mathrm{~m}$ long and is located in the return circuit of the wind tunnel. It is used in the present study to reproduce the properties of a neutral ABL at a reduced scale. To generate the Decaying 'Isotropic' Turbulence, denoted DIT, a turbulence grid is placed at the entrance of themain test section. The turbulence grid is made of metallic square section $25 \mathrm{~mm} \times 25 \mathrm{~mm}$ bars with a mesh size of $100 \mathrm{~mm}$. The reference velocity and turbulence intensity (indicated with index 0 ) are measured at the rotor disc location, but in absence of it. They are $U_{0}=2.5 \mathrm{~m} / \mathrm{s}$ and $I u_{0}=4 \%$, respectively. $I u$ is defined as the ratio between the standard deviation of streamwise velocity $u^{\prime}$ and its time average $U$. The ratio of standard deviations $v^{\prime} / u^{\prime}$ and $w^{\prime} / u^{\prime}$ are 1.06 and 1.03, respectively. This indicates the isotropy of the approach flow.

The flow in the return circuit is tuned by pre-defined installations in the flow processing unit upstream the test section resulting in a modelled moder- 
ately rough $\mathrm{ABL}$ (Roughness length $z_{0}=0.03 \mathrm{~mm}$ in wind tunnel scale, power law coefficient $\alpha=0.14$ ). The geometric scale of $1: 300$ is deduced from the best fit between wind tunnel data and literature about neutral ABL regarding the expected roughness length range, turbulence intensity profiles and integral length scale profiles $[8,9,17,20]$. At $\mathrm{z}=300 \mathrm{~mm}$ (hub height), the upstream mean velocity is $U_{0}=2.5 \mathrm{~m} / \mathrm{s}$ and the turbulence intensity is $I u_{0}=13 \%$ (Figure 2 ). According to experimental limitations, the velocity profile is measured up to $900 \mathrm{~mm}$ above ground. Results show that the boundary layer limit is no yet reached at this altitude.

The 3-blade rotating wind turbine (Fig. 3) has a diameter $D=416 \mathrm{~mm}$, its rotation is controlled and its tip-speed-ratio is fixed to $T S R=5.8$. the work of Sunada et al. [18] has shown that at such low chord Reynolds numbers, standard aerofoil profiles do not behave as they do at high Reynolds numbers. They also showed that thin plate aerofoils behave more like aerofoils at high Reynolds number, except that they stall at a lower lift coefficient. Consequently, the blades were designed according to this statement [14].

The thrust coefficient $C_{T}$ had been experimentally assessed at $C_{T}=0.5$ in a uniform inflow, using the global momentum theory. Knowing the velocity deficit distribution downstream of the rotor $U_{0}-\bar{U}(r)$, the axial force acting on the rotor is obtained by integrating the momentum flux crossing a reference surface :

$$
F_{a x}=2 \pi \int_{0}^{\infty} \rho \bar{U}(r)\left(U_{0}-\bar{U}(r)\right) r d r
$$

The thrust coefficient is then defined as:

$$
C_{T}=\frac{F_{a x}}{\frac{1}{2} \rho U_{0}^{2} S_{D}}
$$

Pascheke and Hancock [14] presented the velocity deficit and turbulence intensity distributions downstream of this rotating wind turbine located in modelled onshore and offshore boundary layers. The values of maximum velocity deficit (40 to $50 \%$ of the mean hub velocity) are representative of full scale wind turbine wakes and are in agreement with literature $[21,24,22]$. Since the wake diffusion is dependent of the boundary layer conditions, it is difficult de precisely compare the wake evolution obtained in the latter references. Nevertheless, the trend is in agreement, showing some self-similar profiles for the velocity deficit and the turbulence intensity in the far wake (from $x / D=3$ to 5 , depending on the terrain roughness and/or thermal stability).

A porous disc made of metallic mesh (Fig. 4) is designed in order to reproduce the same velocity deficit at $x=0.5 D$ downstream of the disc as downstream of the 3-blade rotating wind turbine. Even if the velocity deficit was the only desing criterion, it was nevertheless expected that, if both velocity deficits fitted, the turbulence intensity profiles were similar in areas where the turbulence production is due to velocity gradients (external wake boundaries and hub). On 
the other hand, areas where the turbulence production is due to the grid for the porous disc or to the blade transit for the rotating wind turbine, it is expected to have discrepancies at this short distance downstream of the rotor discs. One of the main reasons of choosing meshes to reproduce the actuator disc at reduced scale in a wind tunnel is that the porous disc can be considered as a grid turbulence generator. This set-up is known to be Reynolds number independent [7]. The porous disc has the same diameter as the wind turbine, the mesh has a solidity of $45 \%$ and a circle of different solidity (35\%) of diameter $0.2 D$ fixed at the center of the main disc. The choice of solidities was driven by a previous parametrical study [2]. The disc is fixed on a mast to be located at the same height as the wind turbine rotor.

$\mathrm{x}, \mathrm{y}$ and $\mathrm{z}$ coordinate origins are located at the rotor center. $\mathrm{x}$ is the streamwise direction, $\mathrm{y}$ is the transverse one and $\mathrm{z}$, the vertical one.

The difference of the static pressure in the flow, $\Delta P_{s}$, is measured as the difference of the static pressure in the wake flow of the rotor disc, $P_{s}$, and the static pressure of the free undisturbed flow, $P_{\text {ext }}$, at least $1 \mathrm{~m}$ above the rotor disc $\left(\Delta P_{s}=P_{s}-P_{\text {ext }}\right)$. Each static pressure is measured through the static branch of a Pitot tube and the static pressure difference $\Delta P_{s}$ is measured with

a DRUCK 0-25 Pa differential pressure transducer with an acquisition time of $180 s$. The $3 \mathrm{D}$ flow properties are measured from $x=0.5 D$ to $3 D$ downstream of the wind turbine with a triple-sensor gold-plated wire probe (Dantec 55P91). It is controlled with a Dantec Streamline CTA system, the sampling frequency is fixed to $6 \mathrm{kHz}$ and the acquisition time to $180 \mathrm{~s}$. The used triple-sensor wire probe is able to measure a velocity vector with an acceptance cone of 70.4 . According to our measurements, the maximal angle between the streamwise direction and the velocity vector is obtained in DIT conditions at $x / D=0.5$, where the rotational momentum is the highest $\left(W / U_{0}=0.18\right.$ and $\left.U / U_{0}=0.55\right)$ and is assessed to a measurement cone of 36 on average, and 62 instantaneously (assuming a maximal instantaneous velocity of $\left.(W+W r m s) / U_{0}=0.33\right)$. The root-mean-square errors of the measured velocity statistics were assessed for the ABL configuration at the worst location (i.e. the worst case: highest turbulence intensity $27 \%$ and largest integral scales $0.5 \mathrm{~m}$ ). For a $95 \%$ confidence interval, $3.3 \%$ error is obtained for the mean velocity, $8.7 \%$ for the root-mean-square velocity, $30 \%$ for the skewness and $121 \%$ for the Kurtosis [4]. These statistical errors are reduced to $0.7 \%, 3.3 \%, 11.3 \%$ and $45.3 \%$, respectively, for the DIT configuration at the worst location.

\section{Results and discussion}

\subsection{Static pressure evolution}

The static pressure coefficient $\left(C_{p}=\left(P_{s}-P_{\text {ext }}\right) /\left(0.5 \rho U_{0}^{2}\right)\right)$ evolution downstream of both rotor discs can be deduced from the static pressure measurements mentioned in the previous part. Figure 5 shows this information at two different transverse locations : at the center $(y / D=z / D=0)$ and at half radius 
$(y / D=0.25, z / D=0)$ of the rotor discs for DIT flow conditions. According to the uniform approach flow, axisymmetry is assumed. The trends and the levels of all curves are similar. The pressure coefficients are negative close to the rotor discs, due to the global energy extraction, tends to zero up to $x / D=1.5$, and stays around zero further downstream. This proofs that the pressure recovery is reached at this distance. For the porous disc, the static pressure coefficient evolution along the $\mathrm{x}$ axis is similar for both transverse locations except very close to the disc, where the pressure loss is slightly lower at the center than at half radius. It illustrates that the energy extraction is lower at the center since the local mesh solidity is lower. On the other hand, this discrepancy disappears very quickly. Making an extrapolation of the $C_{p}$ to $x / D=0$, giving $C_{p}$ of about -0.26 , actuator disc theory implies a $C_{T}$ of 0.59 . A $C_{T}$ of 0.5 would require $C_{p}$ to be -0.23 . Nevertheless, according to the measurement uncertainty due to the use of a static pressure probe in an unsteady and turbulent flow, this result can be considered as acceptable.

For the wind turbine, the pressure loss is higher at the center than at half radius. It is possibly due to the hub wake effect. At the center, the pressure recovery needs consequentely slightly longer distance to be reached . To conclude on this part, the pressure recovery downstream the porous disc and the wind turbine is very similar and is reached at $x / D=1.5$. It is a first step towards the proof of similarity between the wind turbine and the porous disc wake. Additionally, it confirms that the assumption of pressure recovery that is implicitely used in the computation of the thrust coefficient through the use of global momentum theory is valid.

\subsection{Velocity statistic comparison}

Velocity statistics (time-mean, RMS, skewness and Kurtosis) are compared. Figures 6 and 7 show the dimensionless streamwise mean velocity deficit $\left(\left(U_{0}-\right.\right.$ $\left.U(y)) / U_{0}\right)$ profiles versus the spanwise direction $y / D$, at $x / D=0.5$ and 3 downstream of the porous disc and of the wind turbine. As seen on Figures, the porous disc has been correctly designed since since a sufficient similarity between both wakes is established at $\mathrm{x} / \mathrm{D}=0.5 x / D=0.5$. Indeed, the velocity deficit distribution within the wakes is slightly different since the disc porosity is uniform, but the discrepancies do not distort too much the velocity gradients at the wake edges. Farther downstream at $x / D=3$, the wakes, which freely develop, become completely similar for both inflow conditions. Figures 8 and 9 show the streamwise turbulence intensity profiles versus the spanwise direction $y / D$, at $x / D=0.5$ and 3 downstream of the porous disc and of the wind turbine. Regarding the turbulence intensity profiles, it is clear that they show some differences at $x / D=0.5$ due to discrepancies in turbulence production. In the wake of the porous disc, the turbulence production is confined to the edges of the wake, in the annular shear layer due to the existence of a velocity deficit. The mesh itself does not generate significant added turbulence.

A production of turbulence is also visible at the location where the disc porosity changes, due to the associated velocity gradient. In the wake of the wind 
turbine, these latter sources are visible but the turbulence from the wakes of the blades is also noticeable in the middle part of the wake. On the other hand, these discrepancies in turbulence intensity distribution between both wakes have vanished at $x / D=3$ due to the turbulence diffusion. They are even totally suppressed in the ABL inflow conditions.

The measurement locations were rather close from the rotor discs (maximum $3 \mathrm{D}$ downstream) and did not enable us to characterize whether the wake recovery is faster in higher turbulent configurations (as shown in [22]).Nevertheless, the velocity deficit and turbulence intensity profiles present the typical Gaussiantype distribution representative of the far-wake definition only for the higher turbulent configuration (ABL).

Higher-order statistics, as Skewness and Kurtosis of streamwise velocity fluctuations, are also compared on Figures 10, 11, 12 and 13. In DIT conditions, the probability density function of streamwise velocity fluctuations is expected to be of Gaussian type (skewness equal to zero and Kurtosis equal to 3). Figures 10 and 12 validate these expectations out of the wake disturbance area. In ABL flow conditions, the vertical gradient of the streamwise velocity modifies the turbulence properties, which are not described anymore by a Gaussian-type PDF function and lead to different skewness and kurtosis values than 0 and 3, respectively, even in undisturbed areas (see Figs 11 and 13. In both inflow configurations, in the near-wake, the skewness and Kustosis values vary very rapidly in strong velocity gradient areas and trends are relatively differents for porous disc and the wind turbine. On the other hand, at $x / D=3$, the trends are very similar in all configurations, and discrepancies disappear almost entirely in ABL flow conditions.

The last check concerns the streamwise integral length scales $L u_{x}$ (Figures 14 and 15). They were calculated by integrating the autocorrelation function of the streamwise velocity time series according to the time delay, up to the first zero crossing. It is important to notice that the order of magnitude of integral length scales is completely different according to the inflow conditions (ratio of 10 between the length scales measured at the rotor disc location but in absence of it, in ABL and in DIT conditions). In DIT conditions at $x / D=0.5$, the integral length scale is on average $0.05 \mathrm{~m}$ outside of the wake and is forced to $0.02 m$ by the porous disc mesh within the disc wake.

For the rotating wind turbine, the integral length scale is more scattered but stays at a comparable level. In ABL conditions at $x / D=0.5$, the integral length scales are also scattered but are also comparable for both wind turbine models. It shows that the integral length scale is dominantely driven by the upstream turbulent flow properties rather than by the wind turbine model disturbance. Further downstream, in both inflow conditions, the integral length scales are quite similar, even if values are sligthly smaller within the wake of the rotating wind turbine. Knowing the relative difficulty to interpret the absolute value of the integral length scales in non-isotropic flows, one can conclude that these are not significant discrepancies with respect to the simulation of the wake 
flow behind a wind turbine.

To conclude on this part, it is shown that, in relatively high turbulent inflow conditions (ABL conditions here), no significant difference is visible in the wake description of a porous disc or of a rotating wind turbine, regarding the four first moments of distribution functions of velocity fluctuations (mean, standard deviation, Skewness, Kurtosis) and the integral length scales, at a downstream distance of $x / D=3$. On the other hand, discrepancies still exist at $x / D=3$ in low turbulent inflow conditions (DIT conditions) but are relatively minor so one can assume sufficient similarity between the porous disc and the wind turbine wake.

\subsection{Rotational momentum persistence}

In order to check whether the rotational momentum induced by the rotation of the wind turbine is still visible in the far-wake of the wind turbine, Fig. 16 presents the horizontal profile of the mean dimensionless vertical velocity $\left(W(y) / U_{0}\right)$, downstream of the counter-clockwise rotating wind turbine, for both inflow configurations. It shows that the velocity signature of the rotation of the global wake is visible in the near-wake of the wind turbine but becomes insignificant at $x / D=3$ at hub height for ABL inflow conditions. It shows that, if the ambient turbulence level is high enough, the turbulent diffusion, responsible of the turbulence mixing, destroys the coherence of the rotational flow. These results are consistent with Zhang et al, 2012 [23], who obtained similar conclusions in a neutral incoming boundary layer with a turbulence intensity of $8 \%$ at hub height.

Again, one can conclude that, in relatively high turbulent inflow conditions (ABL conditions here), the rotational momentum generated by the blade rotation is smoothed out in the far wake through turbulent diffusion, eliminating the difference between the wake of a porous disc and of a rotating wind turbine.

\subsection{Tip-vortex signature persistence}

One other remaining question is related to the persistence of the tip-vortex signature in the wake of a wind turbine. The streamwise velocity Power Spectral Density downstream of the wind turbine has been determined across the wake. An example of three different spectra is presented on Fig. 17 for DIT inflow conditions and on Fig. 18 for ABL inflow conditions. Velocity time series are acquired at hub height but at the wake borders $y / D=-0.5, z / D=0$ at two downstream locations. At $x / D=0.5$, two frequency peaks are noticeable at the rotation frequency $f=f_{0}$ (with $f_{0}=10 \mathrm{~Hz}$ ) of the wind turbine, and at $f=3 \times f_{0}$, signature of the transit of each blade. The signature is logically more obvious in low turbulent inflow conditions. Peaks due to electronic noise are visible at $f=500 \mathrm{~Hz}\left(f / f_{0}=50\right)$, they must not be considered in this discussion. The flow range where these peaks are visible is located at wake borders (between $y / D=-0.45$ and $y / D=-0.65$ ), where the tip vortex is actually 
generated and advected [12]. On the other hand, no signature of the tip vortex remains visible at $x / D=3$, even for the low turbulent inflow conditions. These results are consistent with Zhang et al $[23,24]$ works. They showed that the tip vortex signatures are not distinguishable anymore from $x / D=3$ in a neutral or convective incoming boundary layer with a turbulence intensity of $8 \%$ at hub height.

One can conclude that the tip-vortex signature in the downstream flow has disappeared at $x / D=3$ and consequently, that its influence on the farther downstream wake evolution is negligible.

\section{Conclusion}

The properties of the wake behind a three-blade rotating wind turbine and behind a porous disc generating a similar velocity deficit were compared through wind tunnel experiments. The goal was to determine whether the use of a simple model as a porous disc (based on the actuator disc concept) to reproduce the wind turbine far wake is satisfactory. Results have shown that the mean velocity deficit, the streamwise turbulence intensity, the streamwise skewness and Kurtosis, the streamwise integral length scale at the beginning of the farwake $(x / D>3)$ downstream of a wind turbine and of a porous disc are closely similar in high intensity turbulent inflow conditions.Furthermore, the rotational momentum generated by the rotor, as well as the tip vortex signature, were not detectable at the latter distance as far as the ambient turbulence intensity is high enough to contribute to accelerate the turbulent diffusion process. This kind of inflow configuration is representative of real full scale situations. The study tends to prove that modelling the wind turbine through a porous disc is enough as far as the far-wake study in ABL inflow conditions is concerned. On the other hand, the comparison obtained in low turbulence inflow configuration is also acceptable, against all expectation. The simplified actuator disc model seems to be usable to reproduce the far wake also for relatively low turbulence conditions.

\section{References}

[1] Jimenez A., Crespo A., Migoya E., Garcia J., (2007) : Advances of largeeddy simulation of a wind turbine wake, The science of making torque from wind, DOI:10.1088/1742-6596/75/1/012041.

[2] Aubrun S., Devinant P., España G., (2007): Physical modelling of the far wake from wind turbines. Application to wind turbine interactions, Proceedings of the European Wind Energy Conference, Milan, Italy.

[3] Aubrun S., Loyer S., España G., Hayden P., Hancock P. (2010) : Is the actuator disk concept sufficient to model the far-wake of a wind turbine? Proceedings of the ITI2010 Conference on Turbulence, Sept. 19-23 2010, Bertinoro, Italy. 
[4] Benedict L.H, Gould R.D. Towards better uncertainty estimates for turbulence statistics. Experiments in Fluids 1996; 22:129-136.

[5] Builtjes P.J.H., Milborrow D.J., (1980): Modelling of wind turbine arrays, Proceedings of the $3^{\text {rd }}$ Int. Symposium Wind Energy Systems, Copenhagen, Denmark.

[6] Cabezón D., Sanz J., Martí I. Crespo A., (2009): CFD modelling of the interaction between the surface boundary layer and rotor wake, Proceedings of the European Wind Energy Conference, Marseille, France.

[7] Chassaing P, (2000): Turbulence en mécanique des fluides, CEPADUESEDITIONS, Toulouse, France. ISBN 2-85428-483-6.

[8] Counihan J., (1975) : Adiabatic atmospheric boundary layers : a review and analysis of data from the period 1880-1972. emphAtmos. Environ. 9:871-905.

[9] ESDU. Characteristics of atmospheric turbulence near the ground Item No. 85020. 1985.

[10] España G., Aubrun S., Loyer S., Devinant P, (2012) Wind tunnel study of the wake meandering downstream of a modelled wind turbine as an effect of large scale turbulent eddies. J. Wind Eng. Ind. Aerodyn. 101:2433

[11] España G., Aubrun S., Loyer S., Devinant P, (2011) Spatial study of the wake meandering using modelled wind turbines in a wind tunnel Wind Energy 14:923937

[12] Hu H., Yang Z., Sarkar P., (2012): Dynamic wind loads and wake characteristics of a wind turbine model in an atmospheric boundary layer wind, Experiments in Fluids 52:1277-1294

[13] El Kasmi A., Masson C., (2008): An extended $k-\epsilon$ model for turbulent flow through horizontal axis wind turbines, Journal of Wind Engineering and Industrial Aerodynamics 96:103-122.

[14] Pascheke F., Hancock P.E., (2009): Influence of ABL characteristics on wind turbine wakes: surface roughness and stratification, Proceedings of the international workshop Physmod 2009, Brussels, Belgium, pp. J.2.1J.2.8.

[15] Port-Agel F.,Wu Y.T., Lu H., Conzemius R.J., (2011): Large-eddy simulation of atmospheric boundary layer flow through wind turbines and wind farms Journal of Wind Engineering and Industrial Aerodynamics 99/4:154-168

[16] Sanderse B.,van der Pijl S.P. and Koren B., (2011): Review of computational fluid dynamics for wind turbine wake aerodynamics, Wind Energy 14:799819. 
[17] Snyder W.H. Guideline for fluid modelling of atmospheric diffusion. US Environment Protection Agency 1981; EPA-600/8-81-009, p.185.

[18] Sunada S., Sakaguchi A. and Kawachi K., (1997): Airfoil section characteristics at a low Reynolds number, Journal of Fluid Engineering 119:129-135

[19] Troldborg N., Sorensen J.N., Mikkelsen R., (2010): Numerical simulations of wake characteristics of a wind turbine in uniform inflow, Wind Energy 13(1):8699.

[20] VDI-guideline 3793/12. Physical modelling of flow and dispersion processes in the atmospheric boundary layer, application of wind tunnels. Beuth Verlag 2000, Berlin.

[21] Vermeer LJ, Sorensen JN, Crespo A., (2003): Wind turbine wake aerodynamics Progress in Aerospace Sciences 39: 467-510

[22] Wu YT and Port-Agel F., (2012): Atmospheric Turbulence Effects on Wind-Turbine Wakes: An LES Study, Energies, 5:5340-5362

[23] Zhang W, Markfort CD, Port-Agel F., (2012): Near-wake flow structure downwind of a wind turbine in a turbulent boundary layer, Experiments in Fluids, 52: 1219-1235

[24] Zhang W, Markfort CD, Port-Agel F, (2013): Wind turbine wakes in a convective boundary layer : Wind tunnel study. Boundary Layer Meteorology 146: $161: 179$ 


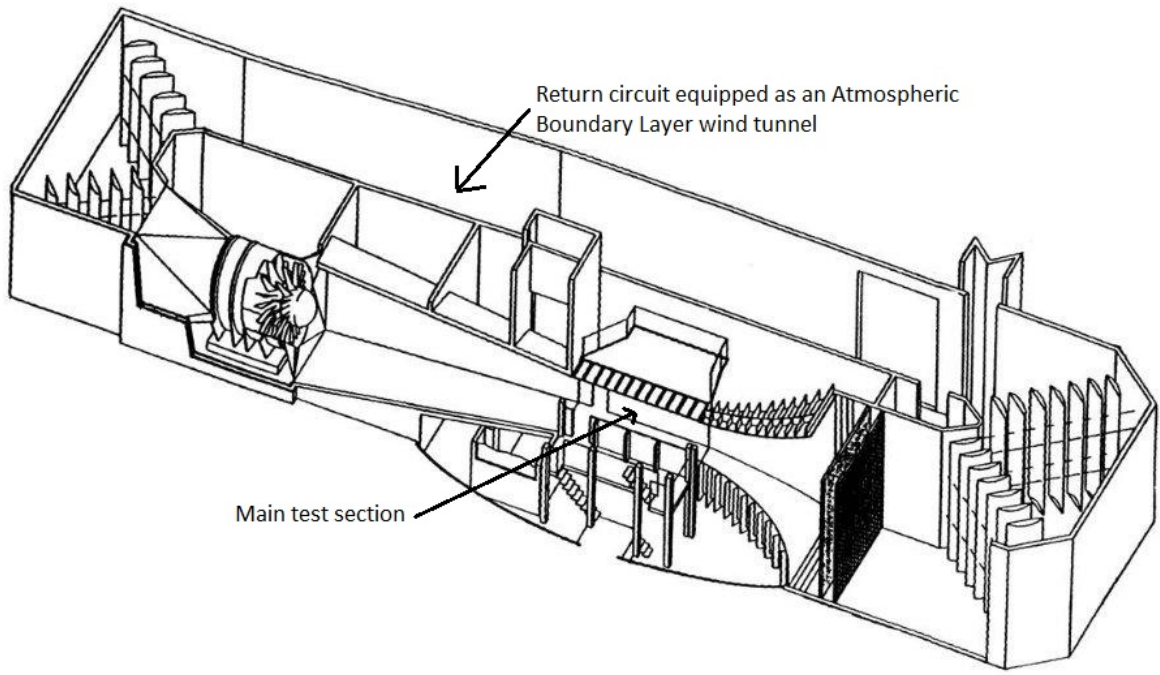

Figure 1: Wind tunnel "Lucien Malavard" ' of the Laboratoire PRISME, University of Orléans, with its two test sections. 

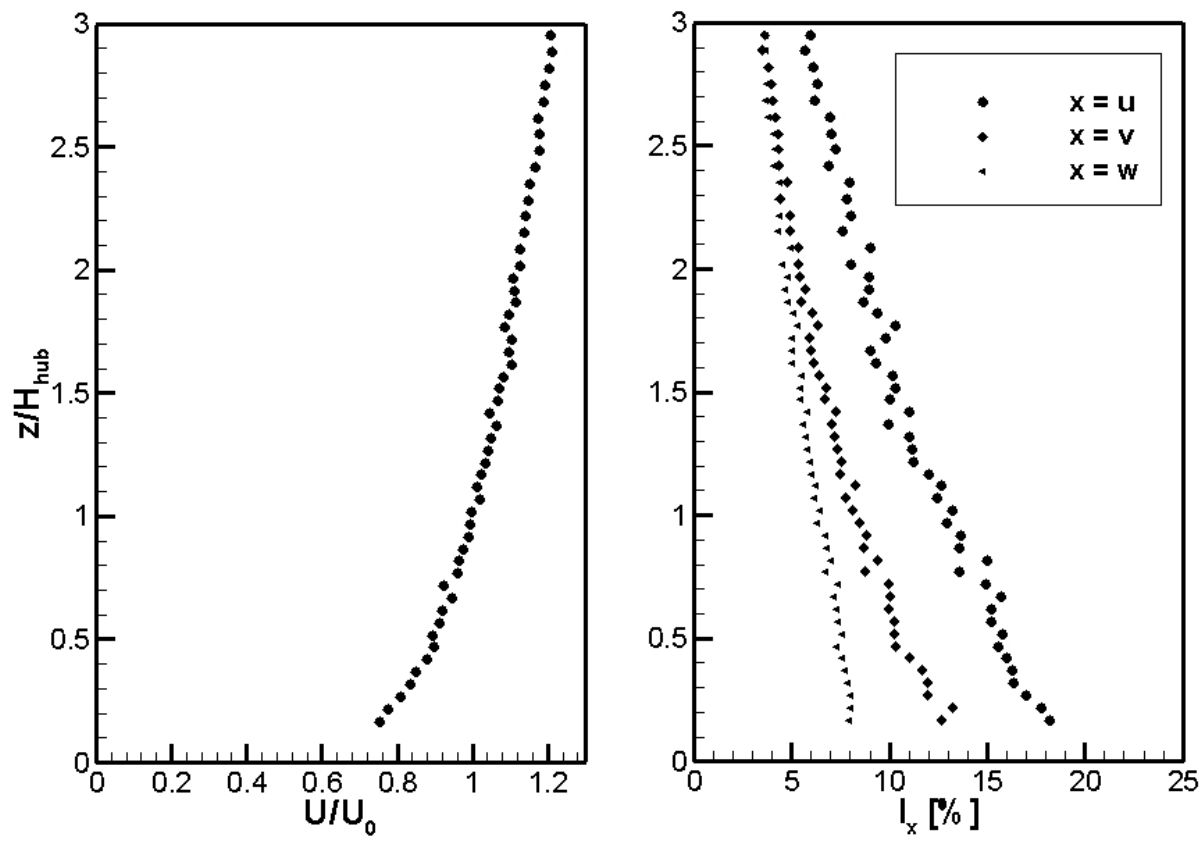

Figure 2: Modelled Atmospheric Boundary Layer properties. Vertical profiles of mean streamwise velocity non-dimensioned with the mean streamwise velocity at hub height (left) and streamwise, crosswise and vertical turbulence intensities (right). 


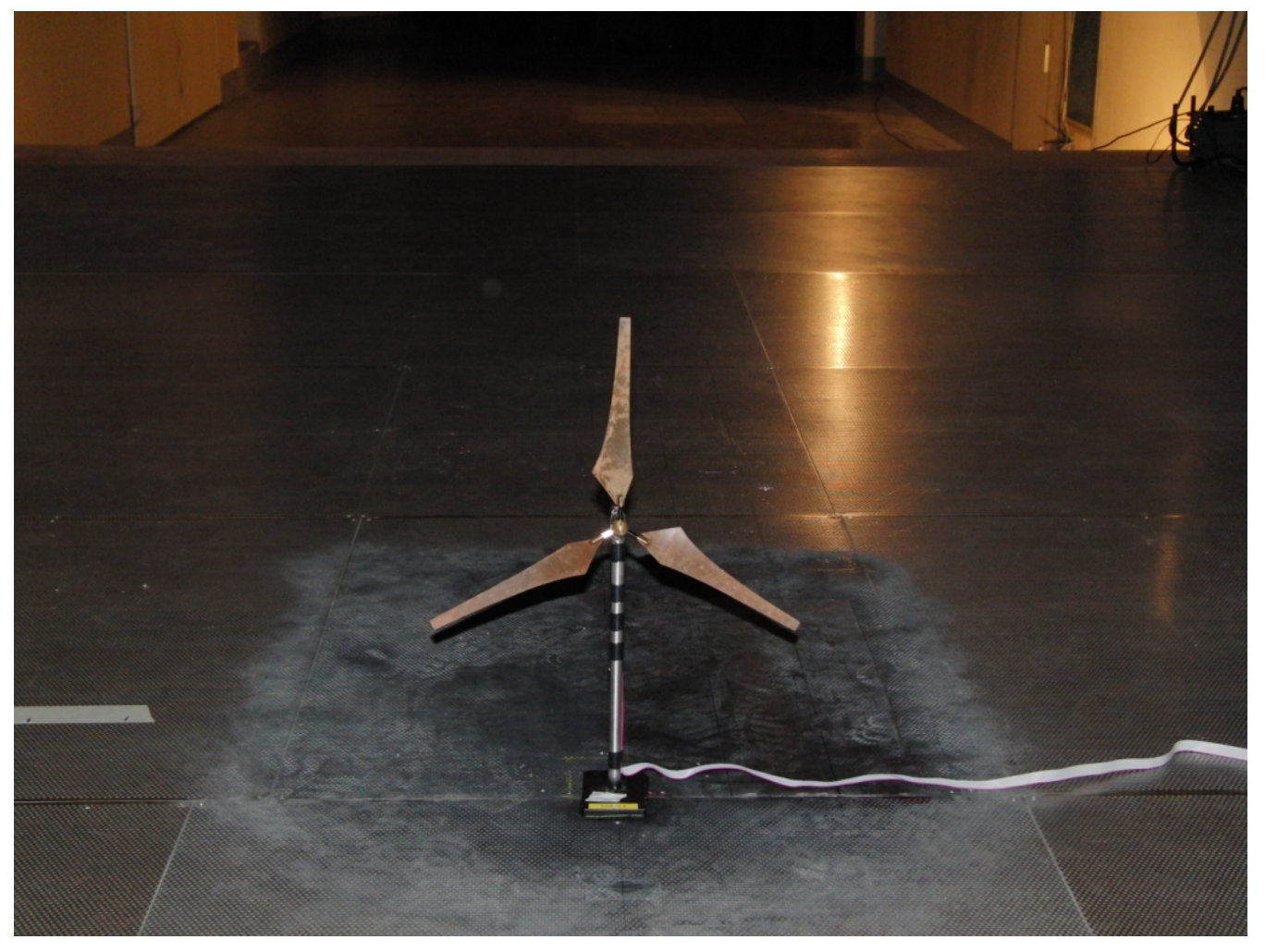

Figure 3: Three-blade rotating wind turbine (EnFlo) 


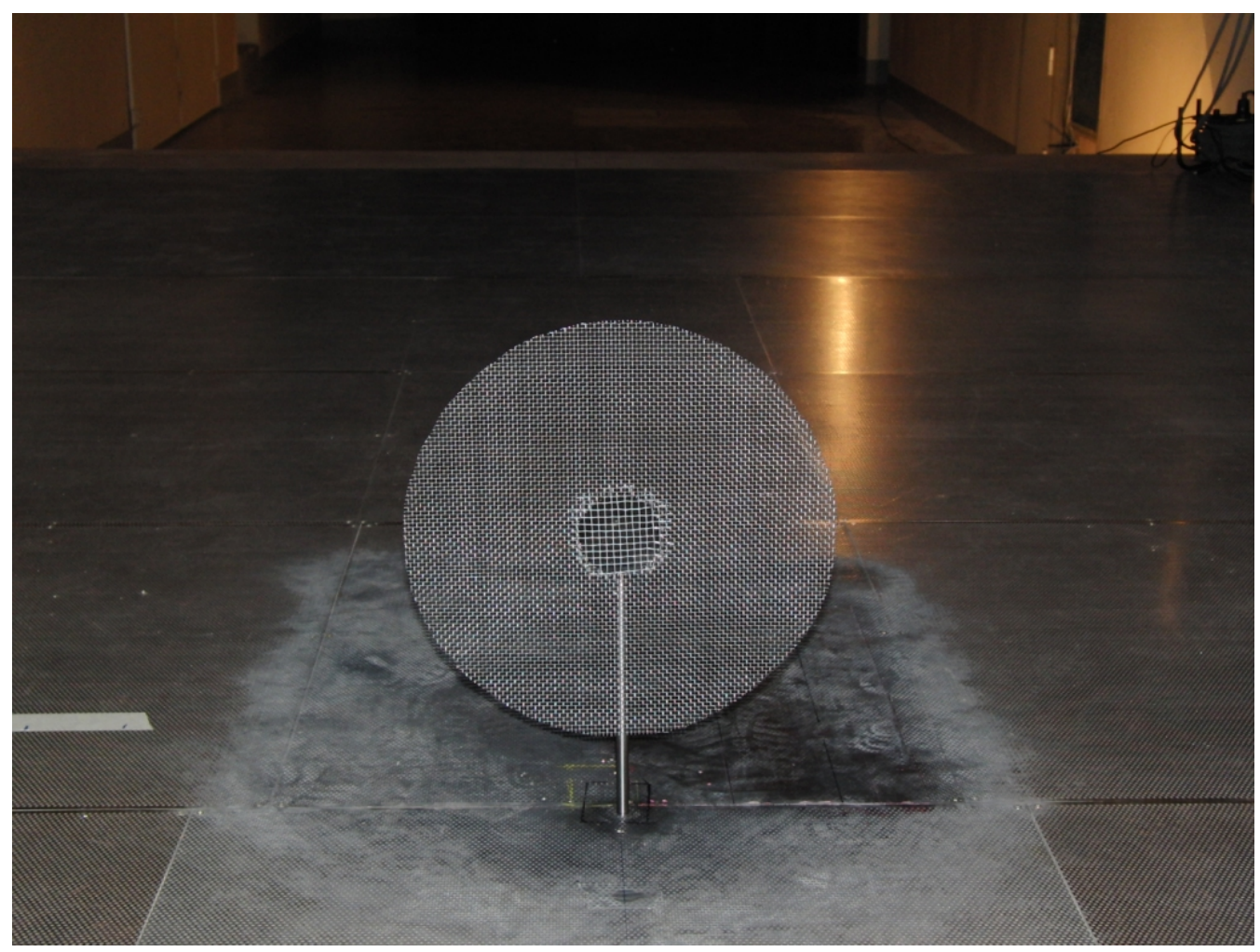

Figure 4: Porous disc generating similar velocity deficit as the rotating wind turbine (PRISME) 


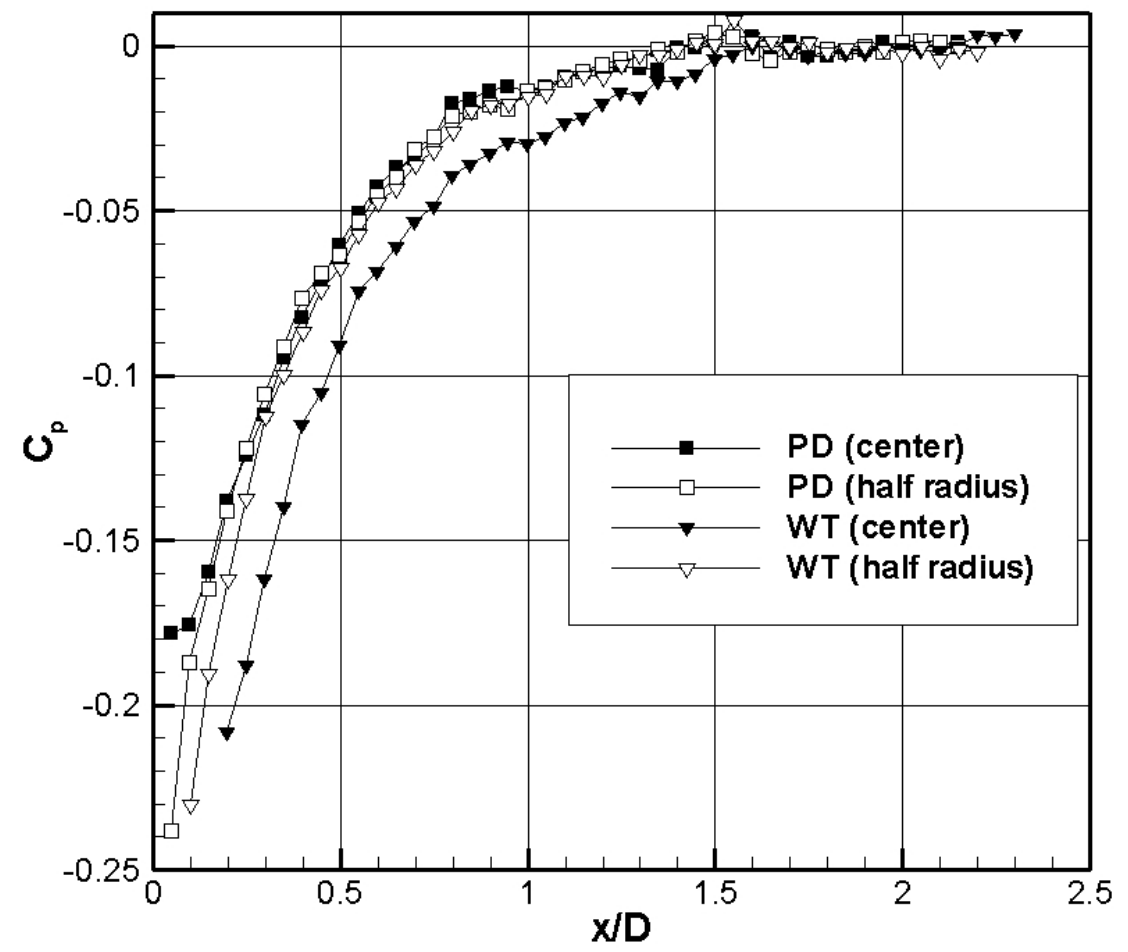

Figure 5: Static Pressure coefficient versus the downstream distance from the porous disc and the wind turbine at two different crosswise locations, along $\mathrm{x}$-axes (centre) and a parallel quart rotor diameter off-centre axis (DIT conditions). 


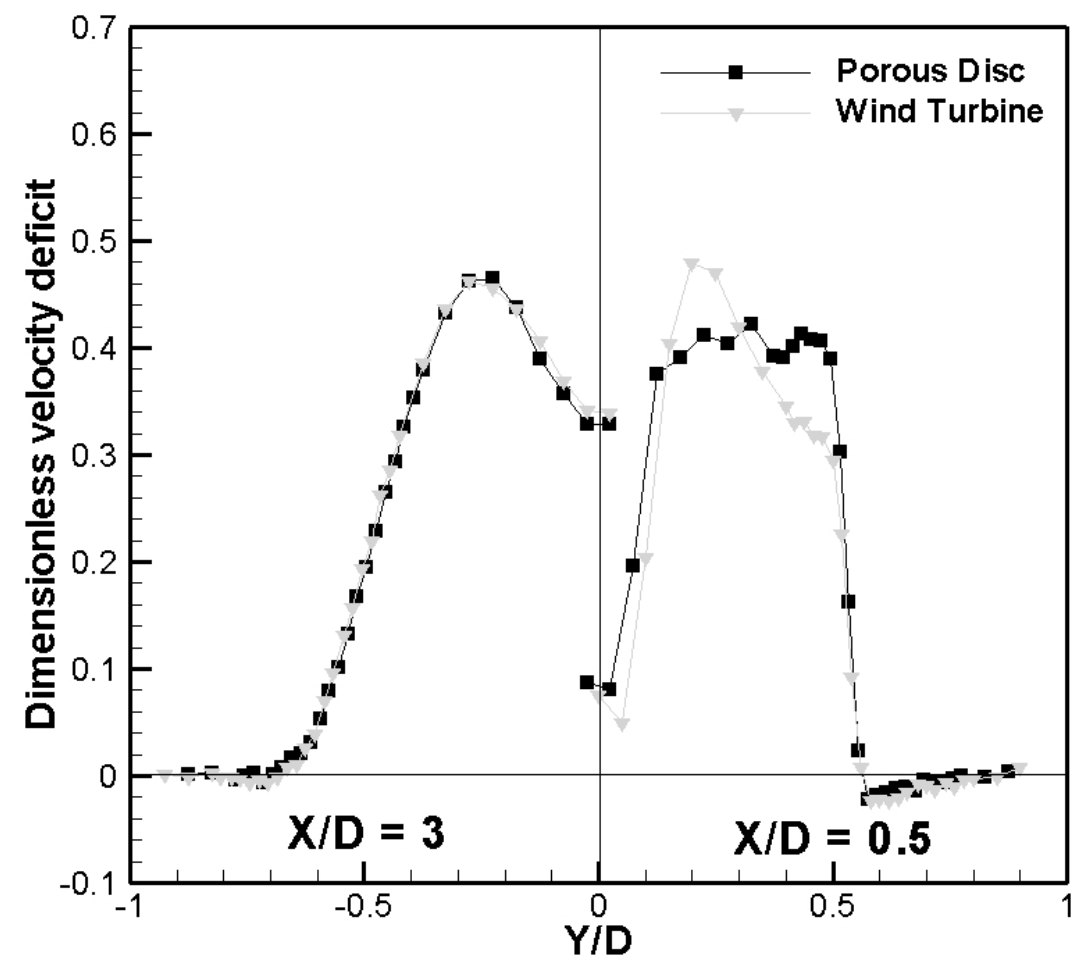

Figure 6: Mean streamwise velocity deficit downstream of the porous disc and the wind turbine (DIT conditions). Left : at $x / D=3$, right : at $x / D=0.5$. 


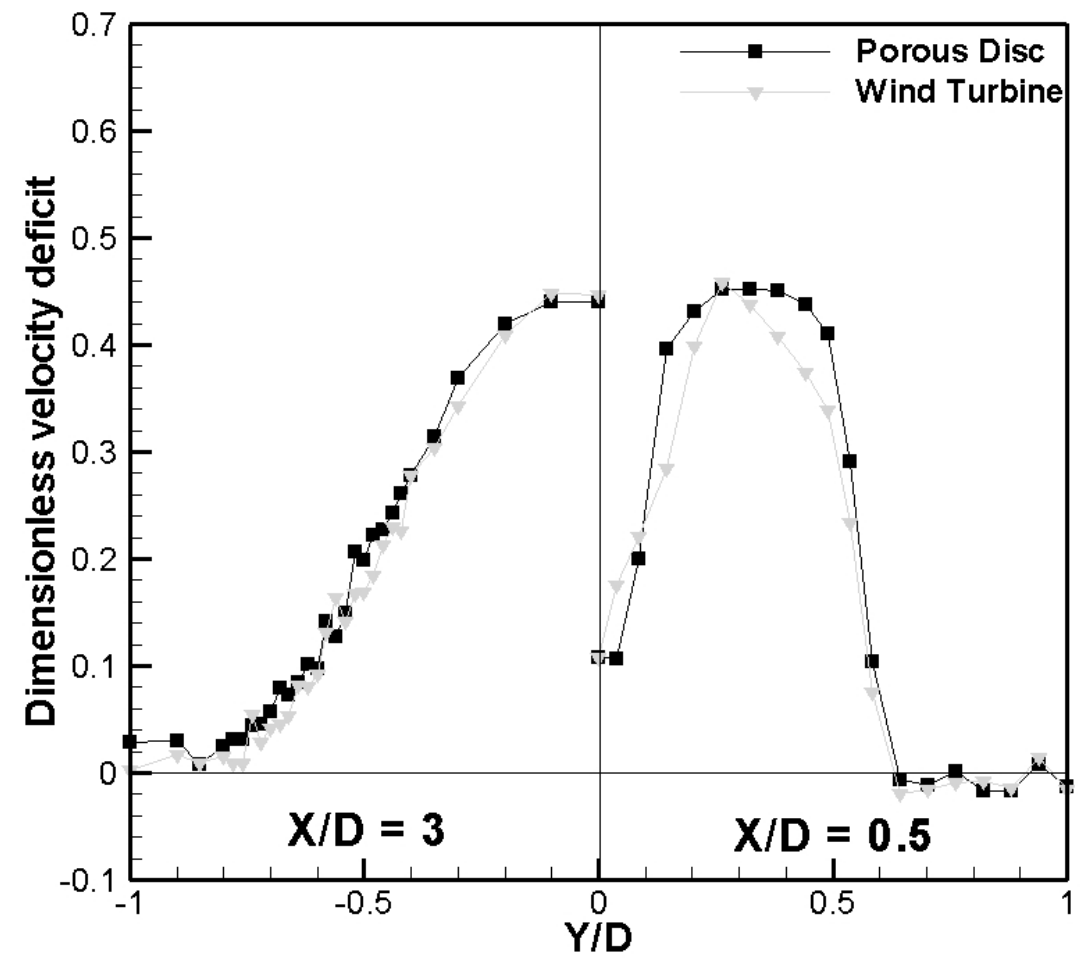

Figure 7: Mean streamwise velocity deficit downstream of the porous disc and the wind turbine (ABL conditions). Left : at $x / D=3$, right : at $x / D=0.5$. 


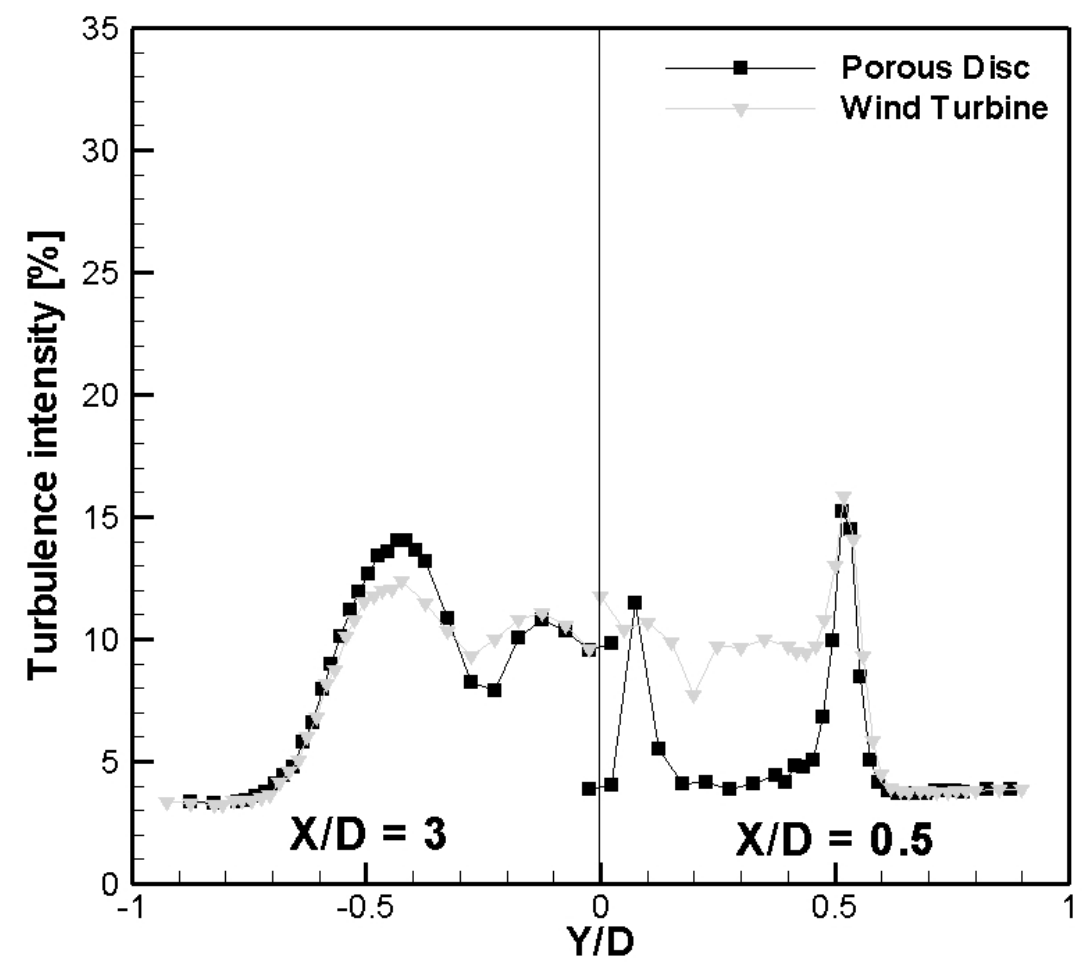

Figure 8: Streamwise turbulence intensity downstream of the porous disc and the wind turbine (DIT conditions). Left : at $x / D=3$, right : at $x / D=0.5$. 


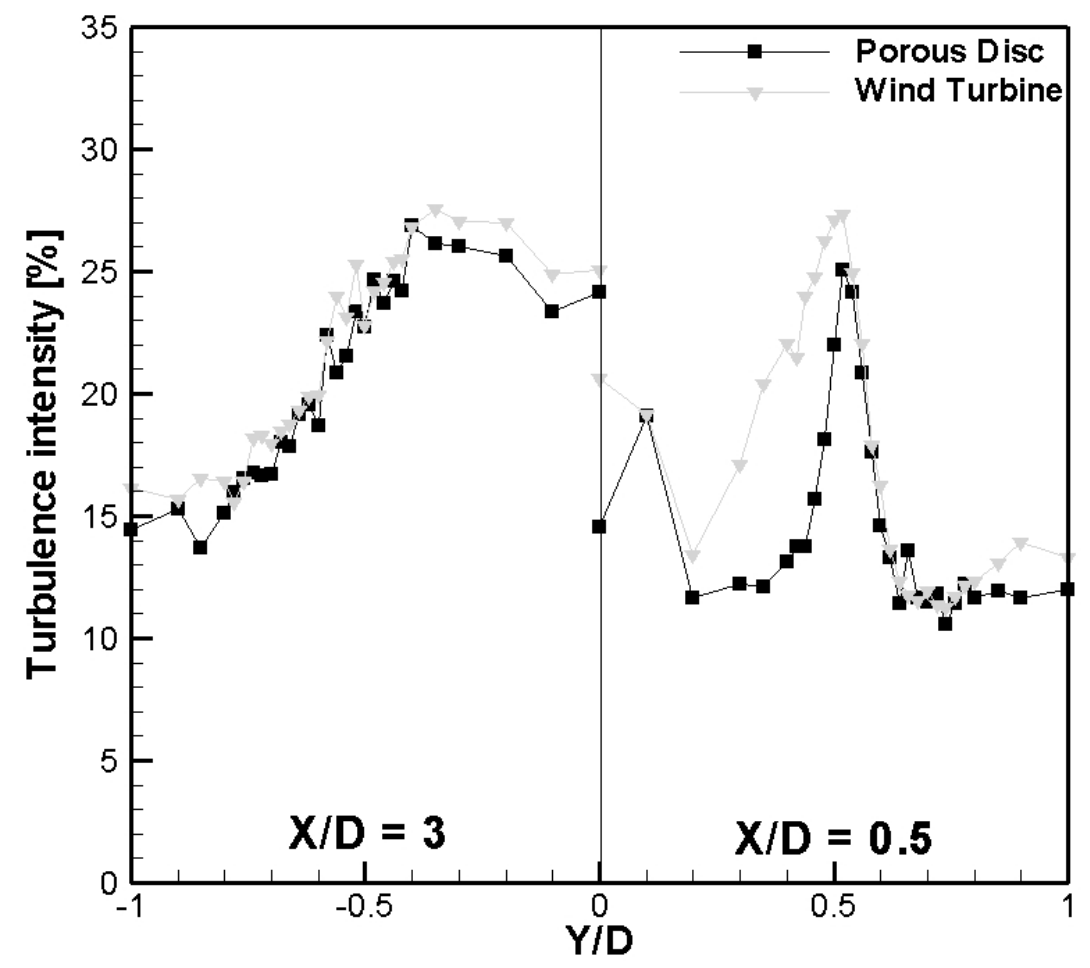

Figure 9: Streamwise turbulence intensity downstream of the porous disc and the wind turbine (ABL conditions). Left : at $x / D=3$, right : at $x / D=0.5$. 


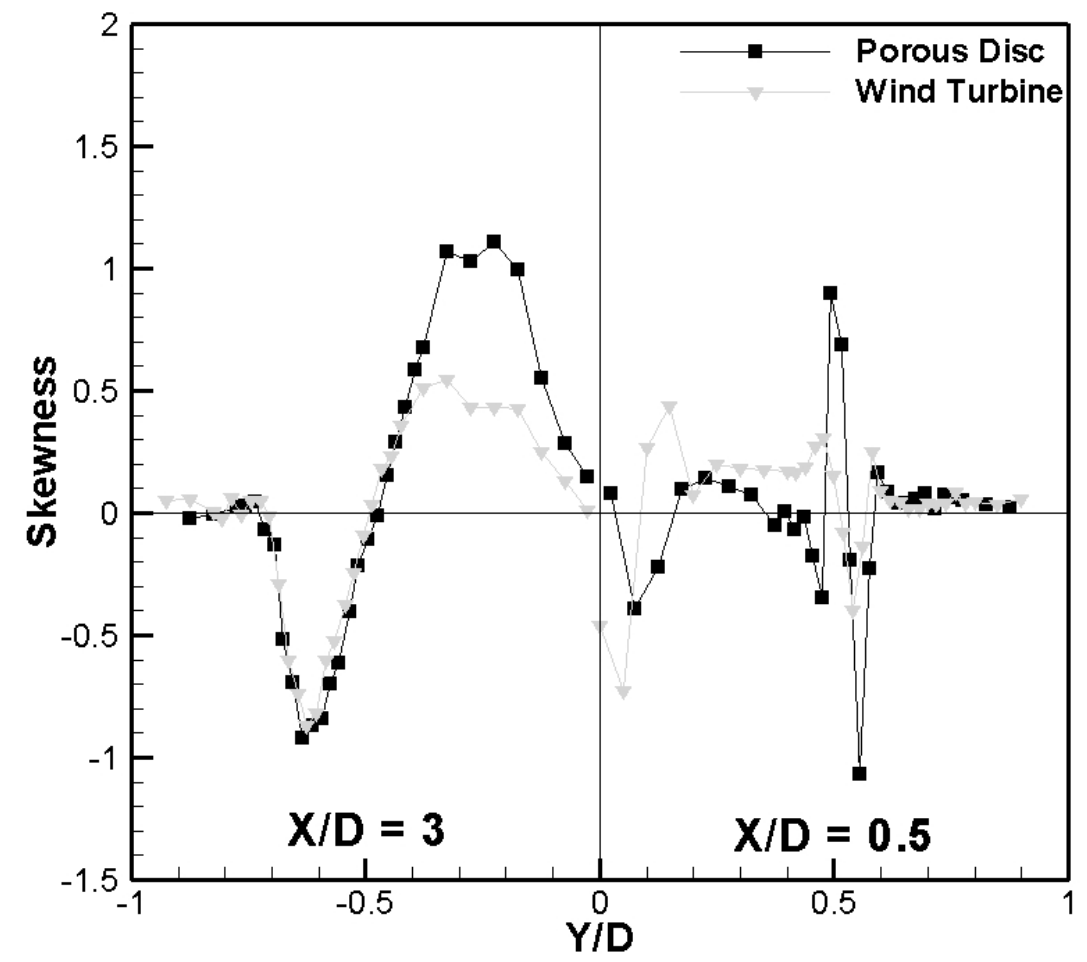

Figure 10: Streamwise skewness downstream of the porous disc and the wind turbine (DIT conditions). Left : at $x / D=3$, right : at $x / D=0.5$. 


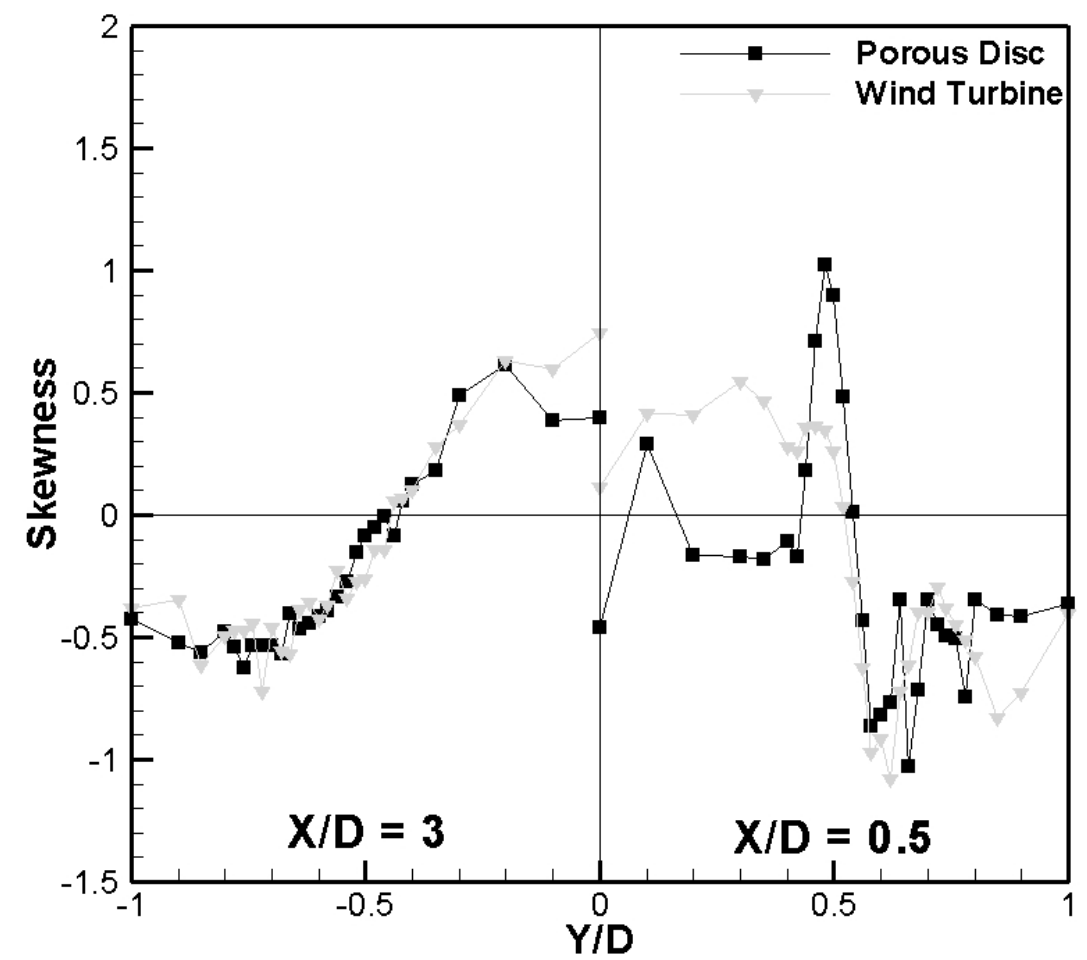

Figure 11: Streamwise skewness downstream of the porous disc and the wind turbine (ABL conditions). Left : at $x / D=3$, right : at $x / D=0.5$. 


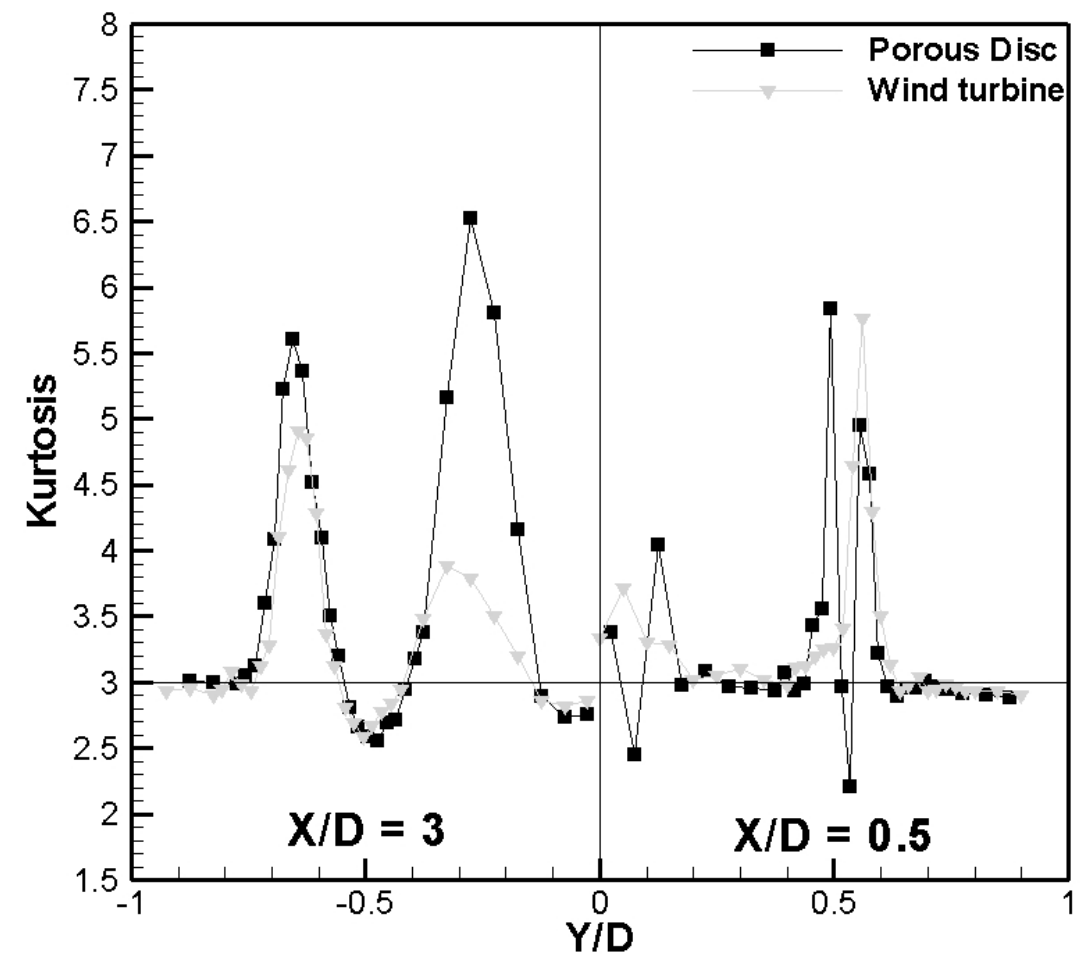

Figure 12: Streamwise Kurtosis downstream of the porous disc and the wind turbine (DIT conditions). Left : at $x / D=3$, right : at $x / D=0.5$. 


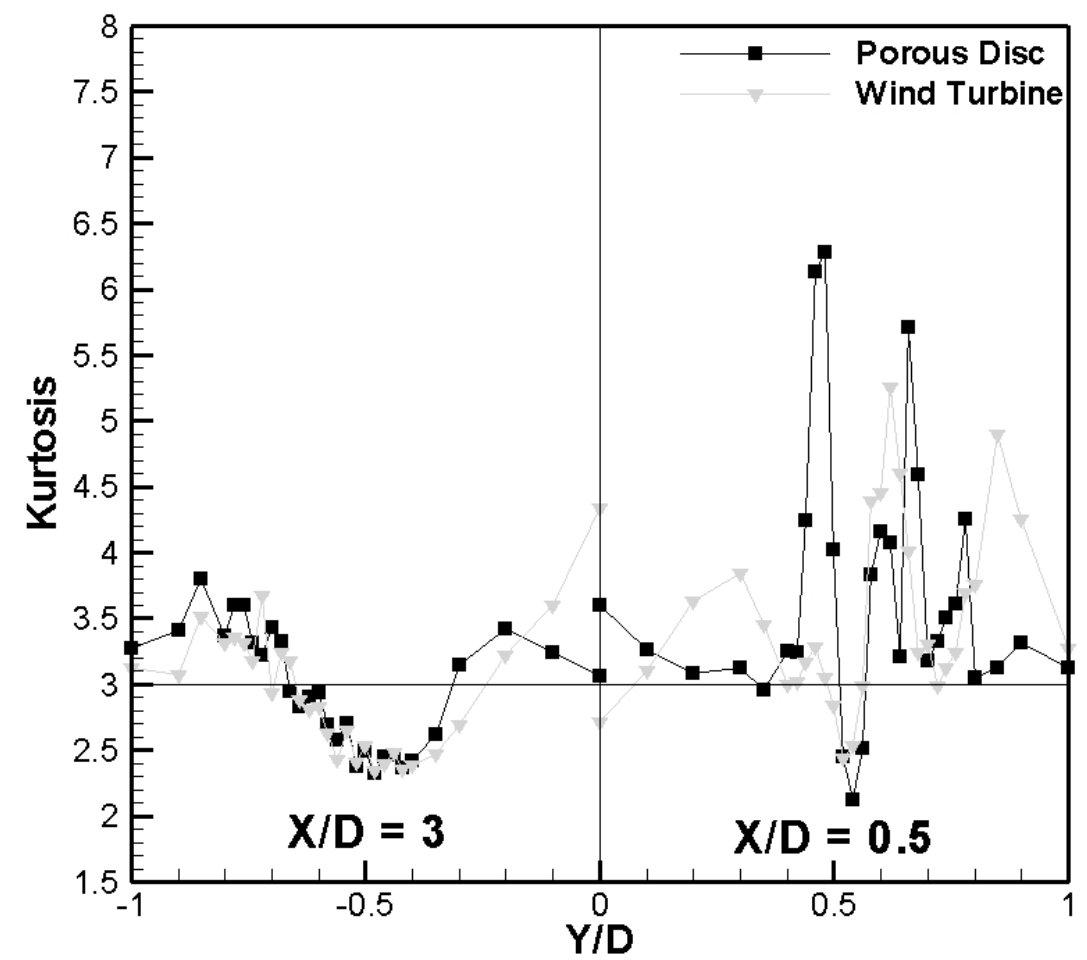

Figure 13: Streamwise Kurtosis downstream of the porous disc and the wind turbine (ABL conditions). Left : at $x / D=3$, right : at $x / D=0.5$. 


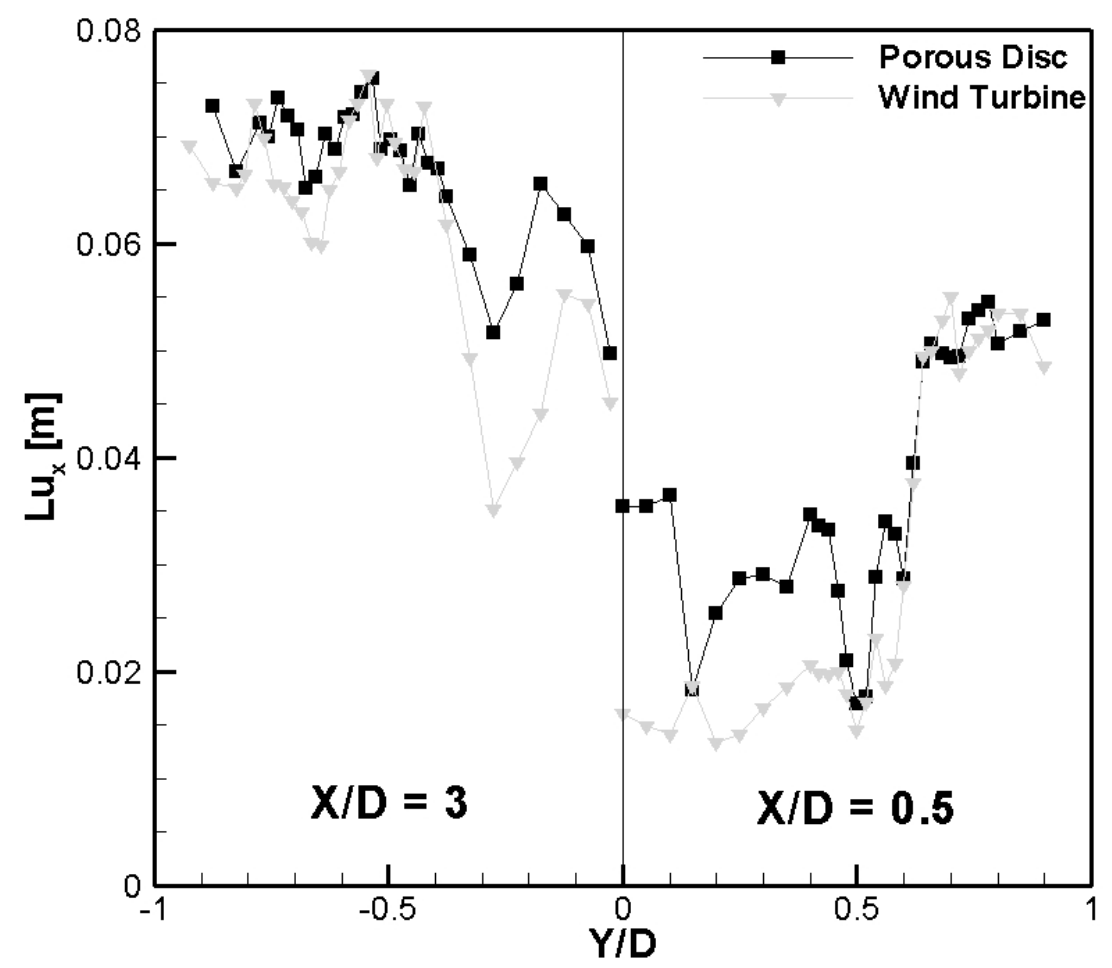

Figure 14: Streamwise integral length scale downstream of the porous disc and the wind turbine (DIT conditions). Left : at $x / D=3$, right : at $x / D=0.5$. 


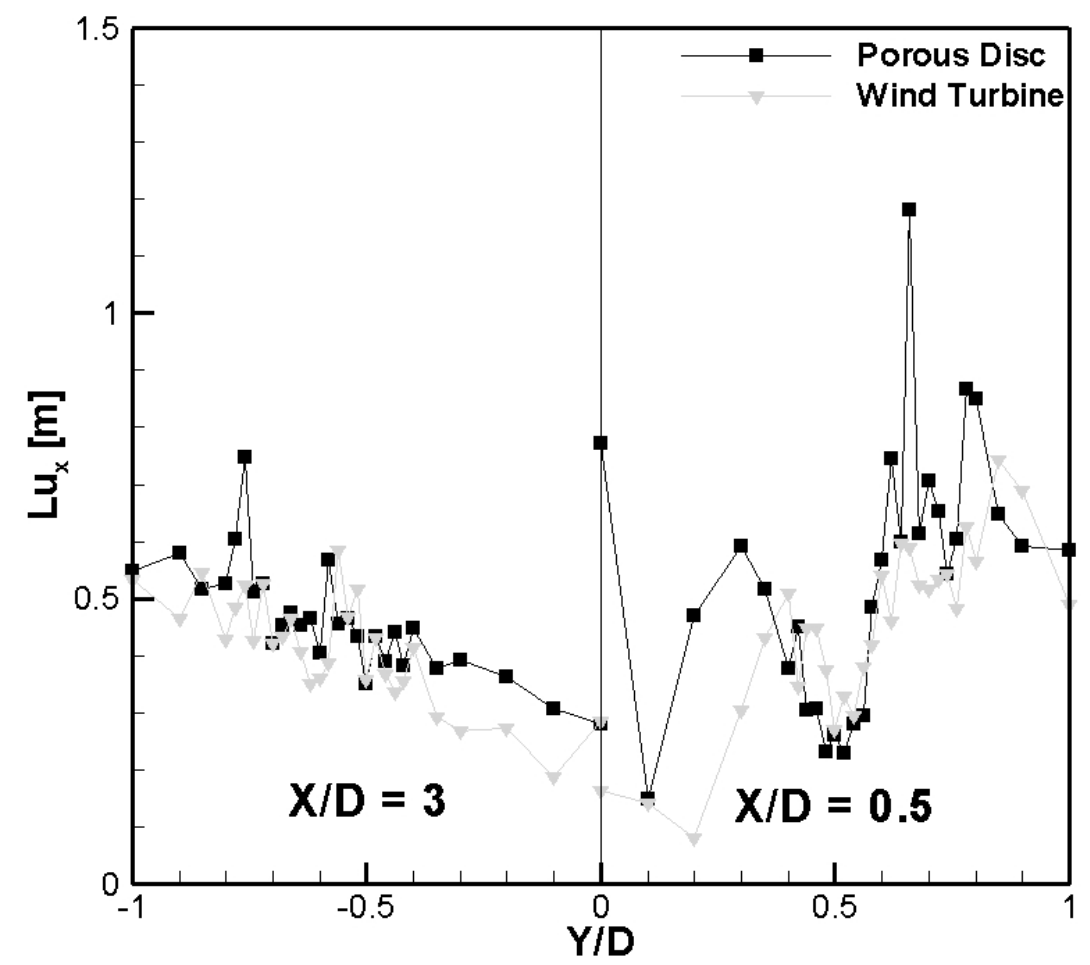

Figure 15: Streamwise integral length scale downstream of the porous disc and the wind turbine (ABL conditions). Left : at $x / D=3$, right : at $x / D=0.5$. 


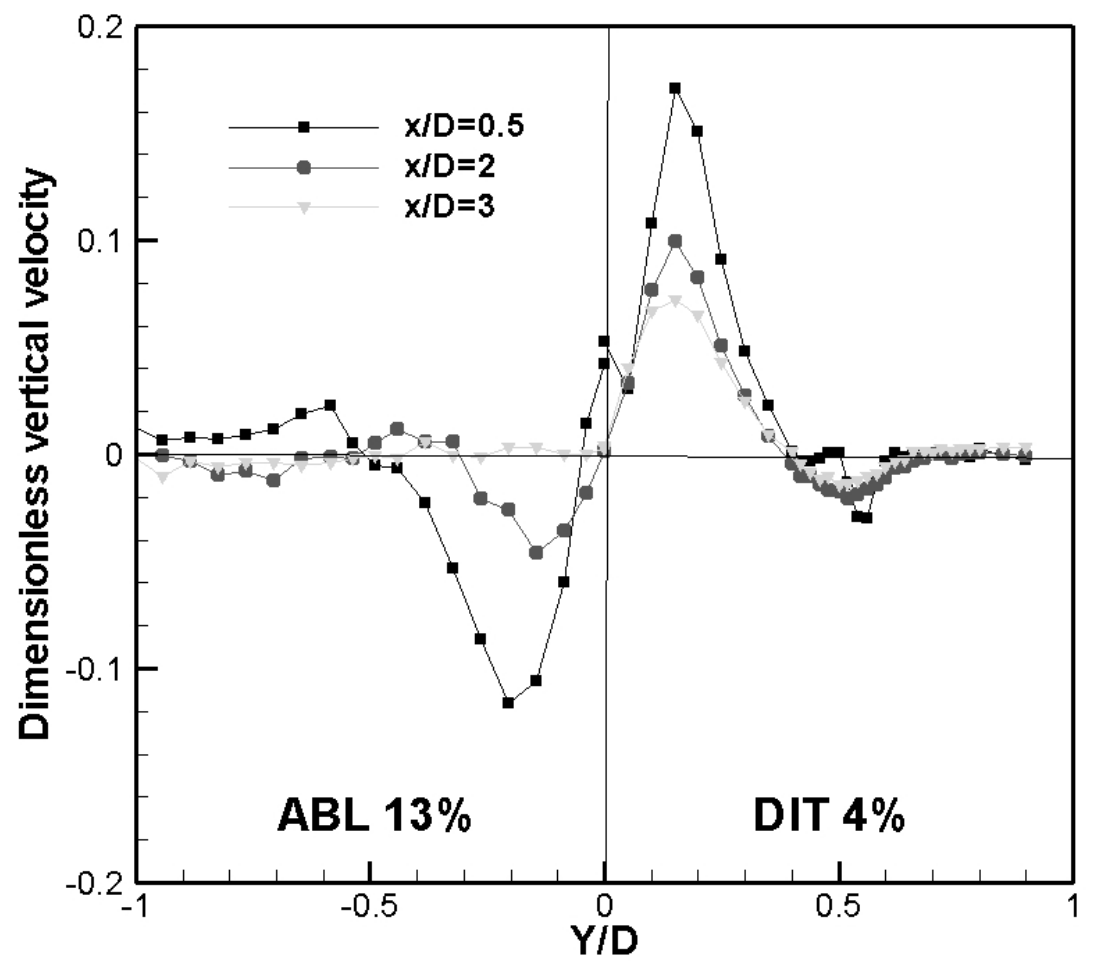

Figure 16: Spanwise profile of the mean vertical velocity downstream of the wind turbine measured at hub height. Left: in ABL inflow conditions (13\% turbulence intensity at hub height), right: in DIT inflow conditions (4\% turbulence intensity). 


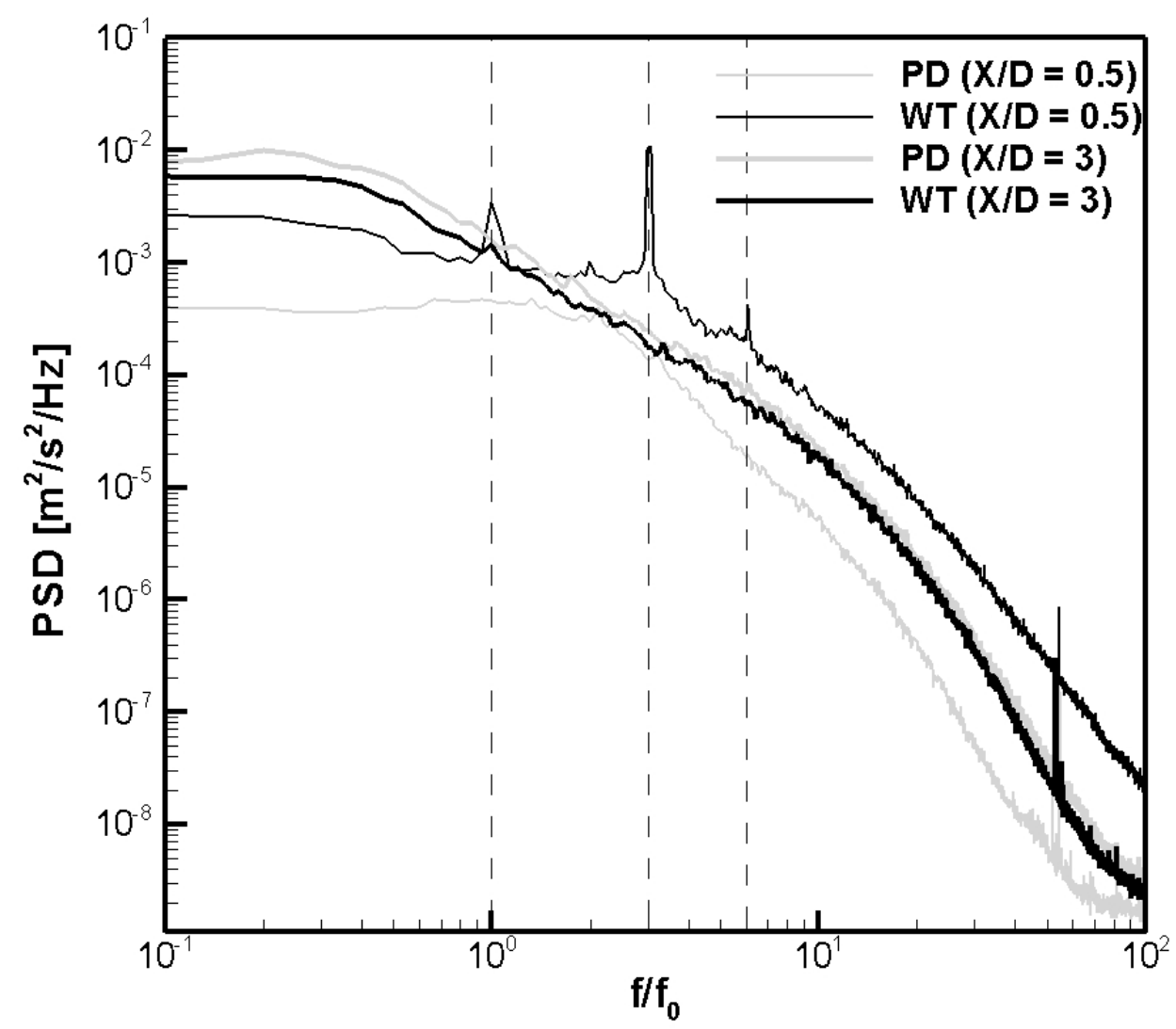

Figure 17: Streamwise velocity PSD at $y / D=-0.5, z / D=0$, downstream of the porous disc and the wind turbine (DIT conditions). 


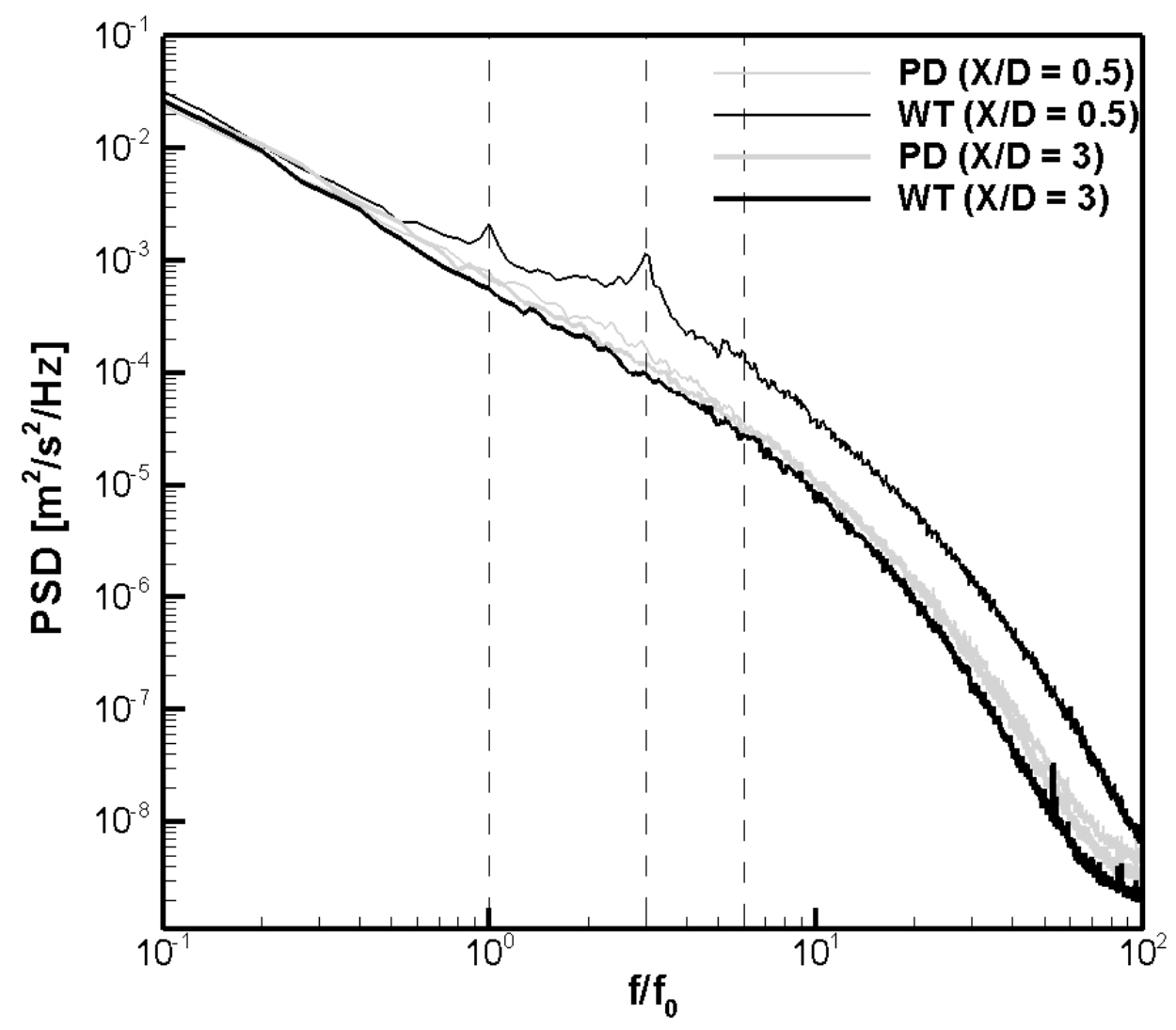

Figure 18: Streamwise velocity PSD at $y / D=-0.5, z / D=0$, downstream of the porous disc and the wind turbine (ABL conditions) 